\title{
Thyroid Gland Oxyphil Cell
}

National Cancer Institute

\section{Source}

National Cancer Institute. Thyroid Gland Oxyphil Cell. NCI Thesaurus. Code C33925.

A large, granular eosinophilic cell derived from thyroid follicular epithelium by accumulation of mitochondria 